\title{
On the Calculation of Moments of Molecular Weight Distribution from Sedimentation Equilibrium Data
}

\author{
Irwin H. Billick, Michael Schulz, ${ }^{1}$ and George H. Weiss ${ }^{2}$ \\ Institute for Materials Research, National Bureau of Standards, Washington, D.C., 20234
}

(October 4, 1966)

\begin{abstract}
In this paper we discuss a technique for calculating moments of polydisperse materials in terms of concentration readings along the cell. The proposed method minimizes dependence on data from the end points where they may be unreliable. An analysis is given of the errors involved in the use of the proposed method when the underlying molecular weight distribution is the Schulz distribution or the lognormal.
\end{abstract}

Key Words: Molecular weight average, molecular weight distributions, moments, polydispersity, polynomial representation, sedimentation equilibrium.

One of the primary functions of a sedimentation equilibrium experiment is to measure the molecular weight of the solute, and in the case of a polydisperse solute, obtain information about the molecular weight distribution. In the latter case, the information is in the form of the first several moments of the distribution. The commonly used methods of data analysis derive these moments from the values of the concentration and its spatial derivatives evaluated at the end points of the solution column [1]. ${ }^{3}$ Methods of evaluating the moments using a point nearer the center of the solution column have been described by Fujita [2] and Adams [3]. However, these techniques require data over the entire range from zero to infinite centrifugal field. Thus far, no practical test of these latter methods has appeared in the literature.

Recently it has been suggested [4] that improved accuracy could be obtained for the moments and hence the molecular weight averages if measurements were made as a function of centrifugal field as the field approaches zero. This latter method again requires some data to be obtained by extrapolation to the meniscus and other data to be obtained by extrapolation to the cell bottom or from a point near the center of the cell. The purpose of this paper is to present a general method of analyzing experimental data in which the end points play a less important role and advantage is taken of the more accurate data obtainable elsewhere in the cell. It will also be shown that the treatment of Osterhoudt and Williams [4] represents a special case of the general treatment presented below. In addition to presenting the method we shall analyze possible errors in the method when the underlying molecular weight distribution is the Schulz [5] distribution or the lognormal distribution [1].

${ }^{1}$ Present address: Department of Physics, M.I.T., Cambridge, Mass.

${ }^{2}$ National Cancer Institute, National Institutes of Health, Bethesda, Md., 20014

${ }^{3}$ Figures in brackets indicate the literature references at the end of this paper.
Method of analysis. In the case of an ideal, noncompressible solution the radial concentration distribution of a single solute species $c_{i}(\zeta)$ of molecular weight $M_{i}$, is given by [2]

$$
\frac{c_{i}(\zeta)}{c_{i}^{0}}=\frac{\lambda M_{i} \exp \left(-\lambda M_{i} \zeta\right)}{1-\exp \left(-\lambda M_{i}\right)}
$$

where $c_{i}^{0}$ is the original concentration before sedimentation and where $\zeta$ is the reduced radial variable given by

$$
\zeta=\frac{b^{2}-r^{2}}{b^{2}-a^{2}}
$$

The distance of the meniscus and bottom of the solution column from the center of rotation are given by $a$ and $b$ respectively, and $r$ is any arbitrary intermediate position. The quantity $\lambda$, is defined by

$$
\lambda=\frac{(1-\bar{v} \rho)\left(b^{2}-a^{2}\right) \omega^{2}}{2 R T}
$$

where the other symbols have their usual definitions [2].

For a solute which is not monodisperse but has a continuous distribution of molecular weights given by some function $f(M)$, the radial distribution of the concentration at sedimentation equilibrium is given by

$$
\frac{c(\zeta)}{c_{0}}=\theta(\zeta)=\int_{0}^{\infty} \frac{\lambda M f(M) e^{-\lambda M \zeta} d M}{1-e^{-\lambda M}}
$$

where $\theta(\zeta)$ is defined by this equation.

One observes that the form of the function given by the right-hand side of eq (1) and contained in the integrand of eq (4) is the same as that of the generating function for the Bernoulli polynomials [6], provided 
that $\lambda M \leqslant 2 \pi$. Thus eq (4) can be rewritten

$\theta(\zeta)=\sum_{n=0}^{\infty} \frac{(-1)^{n}}{n !} B_{n}(\zeta) \lambda^{n} \nu_{n}^{*}+\int_{2 \pi / \lambda}^{\infty} \frac{\lambda M e^{-\lambda .1 / \zeta} f(M) d M}{1-e^{-\lambda .11}}$,

where $\nu_{\|}^{*}$ is the truncated moment

$\nu_{n}^{*}=\int_{0}^{2 \pi / \lambda} M^{\prime \prime} f(M) d M=\left\{\begin{array}{l}M_{u}^{*} \quad, n=1 \\ \left(M_{u} M_{z} M_{z+1} \ldots M_{z+n-2}\right)^{*}, n>1\end{array}\right.$

$M_{w}^{*}$, the truncated weight average molecular weight and so on. Some properties of the polynomials $B_{n}(\zeta)$ are given in reference 6 .

The basis for the method suggested by Osterhoudt and Williams is the replacement of $\theta(\zeta)$ by $\theta^{*}(\zeta)$, defined by

$$
\theta^{*}(\zeta)=\sum_{n=0}^{\infty} \frac{(-1)^{n}}{n !} B_{n}(\zeta) \lambda^{n} \nu_{n}^{*}
$$

i.e., the integral in eq (5) is assumed to be negligible. With this definition, the identity $d B_{n}(\zeta) / d \zeta=n B_{n-1}(\zeta)$, and the particular values $B_{0}(0)=1, B_{1}(0)=-1 / 2$, $B_{\cdot}(0)=1 / 6, \quad B_{3}(0)=0, \quad B_{4}(0)=-1 / 30, \quad B_{5}(0)=0, \ldots$ we readily obtain the result of Osterhoudt and Williams

$\left.\frac{d \theta(\zeta)}{d \zeta}\right|_{\zeta=0}=-\lambda \nu_{1}^{*}-\frac{1}{2} \lambda^{2} \nu_{2}^{*}-\frac{1}{12} \lambda^{3} \nu_{3}^{*}+\frac{1}{120} \lambda^{4} \nu_{4}^{*}+\ldots$

The remaining formulas in reference 4 for $\zeta=1 / 2$ and 1 are derived in similar fashion. However, we can also derive other identities that allow us to use any $\zeta$ values between zero and one thus permitting the use of points that obviate extrapolation and are therefore more reliable. For example, we have

$\theta^{*}\left(\frac{1}{2}-\alpha\right)-\theta^{*}\left(\frac{1}{2}+\alpha\right)=2 \alpha \lambda \nu_{1}^{*}$

$$
+\left(\alpha^{3}-\frac{\alpha}{4}\right) \frac{\lambda^{3} \nu_{3}^{*}}{3}+0\left(\lambda^{5} \nu_{*}^{*}\right)
$$

$\theta^{*}\left(\frac{1}{2}-\alpha\right)+\theta^{*}\left(\frac{1}{2}+\alpha\right)=2+\left(\alpha^{2}-\frac{1}{12}\right) \lambda^{2} \nu_{2}^{*}$

$$
+\left(\alpha^{4}-\frac{\alpha^{2}}{2}+\frac{7}{120}\right) \frac{\lambda^{4} \nu_{4}^{*}}{12}+0\left(\lambda^{6} \nu_{6}^{*}\right),
$$

where $\alpha$ can be chosen arbitrarily. By choosing $\alpha=1 / 4$ we find

$\theta^{*}\left(\frac{1}{4}\right)-\theta^{*}\left(\frac{3}{4}\right)=\frac{\lambda \nu_{1}^{*}}{2}-\frac{1}{64} \lambda^{3} \nu_{3}^{*}+\ldots$

$\theta^{*}\left(\frac{1}{4}\right)+\theta^{*}\left(\frac{3}{4}\right)=2-\frac{\lambda^{2} \nu_{2}^{*}}{48}+\frac{119}{46080} \lambda^{4} \nu_{4}^{*}+\ldots$

or choosing $\alpha=0$,

$$
\theta^{*}\left(\frac{1}{2}\right)=1-\frac{\lambda^{2} \nu_{2}^{*}}{24}+\frac{7}{1440} \lambda^{4} \nu_{4}^{*}+\ldots .
$$

Values of the moments are derived from these relations by taking measurements at several values of $\lambda$ and extrapolating to the dependence at $\lambda=0$.

It is possible to extend these considerations so that a series is obtained, the first term of which is proportional to $\lambda^{r} \nu_{r}$, and in which any number of terms in $\nu_{1}^{*}, \nu_{2}^{*}, \ldots, \nu_{r-1}^{*}, \nu_{r+1}^{*}, \ldots \nu_{r+m}^{*}$ have zero coefficients, by properly choosing points $\zeta_{1}, \zeta_{2}, \ldots, \zeta_{r+m}$, and coefficients $\alpha_{1}, \alpha_{2}, \ldots \alpha_{r+m}$, in linear combination:

$$
\begin{aligned}
F^{*}\left(\zeta_{1}, \zeta_{2}, \ldots .\right. & \left.\zeta_{r+m}\right)=\alpha_{1} \theta^{*}\left(\zeta_{1}\right) \\
& +\alpha_{2} \theta^{*}\left(\zeta_{2}\right)+\ldots+\alpha_{r+m} \theta^{*}\left(\zeta_{r+m}\right) .
\end{aligned}
$$

Given the set of points $\zeta_{1}, \zeta_{2}, \ldots, \zeta_{r+m}$ the $\alpha_{i}$ are chosen so that

$\sum_{j=1}^{r+m} \alpha_{j} B_{k}\left(\zeta_{j}\right)=0, k=1,2, \ldots, r-1, r+1, \ldots r+m$

One of the constants, $\alpha_{1}$, can be set equal to 1 and the ratios $\alpha_{j} / \alpha_{1}$, are determined from eq (13). The points $\zeta_{j}$ can be chosen arbitrarily except for the condition

$$
\sum_{j=1}^{r+m} \alpha_{j} B_{r}\left(\zeta_{j}\right) \neq 0
$$

in order that the coefficient of $\lambda^{r} \nu_{r}^{*}$ be nonzero. As an example we can calculate a formula in which the first nonzero is proportional to $\lambda^{3} \nu_{3}^{*}$, and the second $\lambda^{5} \nu_{5}^{*}$, by choosing

$$
\zeta_{1}=1 / 5, \zeta_{2}=2 / 5, \zeta_{3}=3 / 5, \zeta_{4}=4 / 5
$$

Using the procedure outlined above we find that

$$
\begin{aligned}
-\theta^{*}\left(\frac{1}{5}\right)+3 \theta^{*}\left(\frac{2}{5}\right)-3 \theta^{*}\left(\frac{3}{5}\right) & +\theta^{*}\left(\frac{4}{5}\right) \\
& =\frac{\lambda^{3} \nu_{3}^{*}}{125}-\frac{111 \lambda^{5} \nu_{5}^{*}}{375,000}+\ldots
\end{aligned}
$$


Error analysis. So far we have made the tacit assumption that the observed value $\theta(\zeta)$ is identical to $\theta^{*}(\zeta)$ so that $\nu_{n}^{*}$ can be identified with the desired value $\nu_{n}$. It clearly is not, so that some error analysis is required to set bounds on the validity of the method. Two types of error require investigation. The first is the error in using the observed $\theta(\zeta)$ rather than the required $\theta^{*}(\zeta)$, and the second is in the calculation of values of $\nu_{n}^{*}$ rather than $\nu_{n}$.

Let us denote by $\epsilon(\zeta)$ the difference

$$
\epsilon(\zeta)=\theta(\zeta)-\theta^{*}(\zeta)=\int_{2 \pi / \lambda}^{\infty} \frac{\lambda M e^{-\lambda M \zeta}}{1-e^{-\lambda M}} f(M) d M .
$$

The absoulte error incurred by using values of $\theta^{*}(\zeta)$ rather than $\theta(\zeta)$ can be bounded as follows:

$$
\begin{aligned}
\left|F-F^{*}\right|= & \left|\sum_{j=1}^{r+m} \alpha_{j} \epsilon\left(\zeta_{j}\right)\right| \\
& =\int_{2 \pi / \lambda}^{\infty} \frac{\lambda M f(M)}{1-e^{-\lambda M}}\left|\sum_{j=1}^{r+m} \alpha_{j} e^{-\lambda M \zeta_{j}}\right| d M \\
& \leqslant\left(1-e^{-2 \pi}\right)^{-1} \lambda\left(\nu_{1}-\nu_{1}^{*}\right) \sum_{j=1}^{r+m}\left|\alpha_{j}\right| e^{-2 \pi \zeta_{j}},
\end{aligned}
$$

so that the difference between the observed $F$ and the desired $F^{*}$ is proportional to $\lambda\left(\nu_{1}-\nu_{1}^{*}\right)$. Theoretically one can decrease this bound by choosing the $\zeta_{j}$ close to 1 but this method is limited by observational errors the meniscus. It is interesting to note that if the moments are to be calculated in terms of parameters relating to the derivative $\theta^{\prime}(\zeta)$, as is the case in Osterhoudt and Williams' paper, the bound corresponding to eq (17) contains $\lambda\left(\nu_{2}-\nu_{2}^{*}\right)$ in place of $\lambda\left(\nu_{1}-\nu_{1}^{*}\right)$ and is therefore larger.

For the purpose of illustrating errors in terms of physical quantities we will derive explicitly the formulas for $\lambda\left(\nu_{1}-\nu_{1}^{*}\right)$ and $J_{n}=1-\left(\nu_{n}^{*} / \nu_{n}\right)$ for two common polymer distributions; the Schulz [5] and the lognormal [1]. We consider first the Schulz distribution

$$
f(M)=\frac{a^{\rho+1} M^{\rho}}{\Gamma(\rho+1)} e^{-a M},
$$

where the two adjustable parameters $a$ and $\rho$ can be expressed in terms of $M_{w}$ and $M_{z}$, the weight and $z$ average molecular weights as

$$
\begin{aligned}
& a=\left(M_{z}-M_{w}\right)^{-1} \\
& \rho=\frac{2 M_{w}-M_{z}}{M_{z}-M_{w}} .
\end{aligned}
$$

For this distribution $\lambda\left(\nu_{1}-\nu_{1}^{*}\right)$ can be written

$$
\lambda\left(\nu_{1}-\nu_{1}^{*}\right)=\frac{\lambda}{a \Gamma(\rho+1)} \int_{2 \pi a / \lambda}^{\infty} u^{\rho+1} e^{-u} d u .
$$

We therefore see that the difference depends on the parameters $\rho$ and $\lambda / a$ where

$$
\lambda / a=\lambda\left(M_{z}-M_{w}\right) .
$$

Experiments of the type discussed here can be arranged so that $\lambda M_{w} \approx 1$. Furthermore, the Schulz distribution is a sensible one for polymers only for $\rho$ positive or $2>M_{z} / M_{w}$ hence $\lambda M_{z} \leqslant 2$ and $\lambda / a$ is at most equal to 2 . For $\rho \lambda /(2 \pi a)$ a small number we can approximate the integral in eq (20) by

$$
\begin{aligned}
\int_{2 \pi a / \lambda}^{\infty} u^{\rho+1} e^{-u} d u \sim & \left(\frac{2 \pi a}{\lambda}\right)^{\rho+1} e^{-\frac{2 \pi a}{\lambda}} \\
& {\left[1+(\rho+1)\left(\frac{\lambda}{2 \pi a}\right)+\ldots\right] }
\end{aligned}
$$

so that

$$
\lambda\left(\nu_{1}-\nu_{1}^{*}\right) \sim \frac{(2 \pi)^{\rho+1}}{\Gamma(\rho+1)}\left(\frac{a}{\lambda}\right)^{\rho} e^{-2 \pi a / \lambda} .
$$

In figure 1 we have plotted some representative values of $-\log _{10} \lambda\left(\nu_{1}-\nu_{1}^{*}\right)$ as a function of $\lambda M_{w}$. As can be seen from the figure $\theta(\zeta)$ and $\theta^{*}(\zeta)$ are experimentally indistinguishable when $\lambda M_{w}$ is less than 1 except when $M_{z} / M_{w}$ is greater than 1.75. Even in that case if $\lambda M_{w}$ can be set less than $0.5, \theta(\zeta)$ and $\theta^{*}(\zeta)$ are experimentally indistinguishable.

To estimate the accuracy with which $\nu_{n}^{*}$ approximates to $\nu_{n}$ we calculate the ratios

$$
\begin{aligned}
J_{n}=1-\frac{\nu_{n}^{*}}{\nu_{n}}= & \frac{1}{\Gamma(n+\rho+1)} \int_{2 \pi a / \lambda}^{\infty} u^{\rho+n} e^{-u} d u \\
& \sim\left(\frac{2 \pi a}{\lambda}\right)^{\rho+n} \frac{1}{\Gamma(\rho+n+1)} e^{-2 \pi a / \lambda}
\end{aligned}
$$

In figure 2 we have plotted $-\log _{10} J_{1}$ and $-\log _{10} J_{2}$ as a function of $\lambda M_{w}$ for $M_{z} / M_{w}=1.75$. It is to be noted that both $\nu_{1}$ and $\nu_{2}$ can theoretically be determined to within an error of about $1 \%$ with the present method, provided that $\lambda M_{w}$ is less than 1 .

Another distribution useful in polymer work is the lognormal [1]

$$
f(M)=\frac{1}{\sigma M \sqrt{2 \pi}} \exp \left\{-\frac{1}{2 \sigma^{2}} \ln ^{2}\left(\frac{M}{M_{0}}\right)\right\}
$$

The two adjustable parameters $M_{0}$ and $\sigma$, are related to $M_{w}$ and $M_{z}$ by

$$
\begin{aligned}
M_{0} & =M_{w}\left(M_{w} / M_{z}\right)^{1 / 2} \\
\sigma^{2} & =\ln \left(M_{z} / M_{w}\right) .
\end{aligned}
$$

A straightforward calculation suffices to show that 


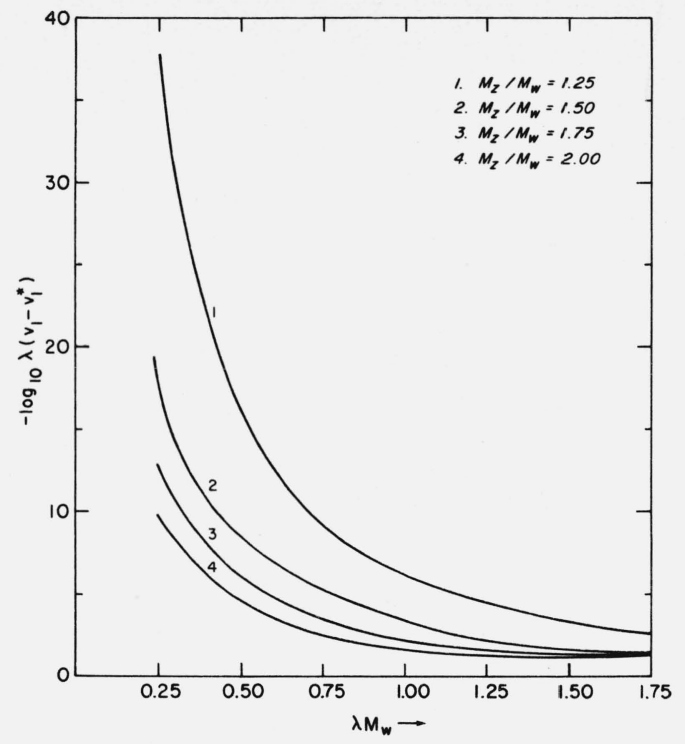

FIGURE 1. Graphs of $-\log _{10} \lambda\left(\nu_{1}-\nu_{1}^{*}\right)$ as a function of $\lambda \mathrm{M}_{\mathrm{w}}$ for different values of $\mathrm{M}_{\mathrm{z}} / \mathrm{M}_{\mathrm{w}}$ for a Schulz distribution.

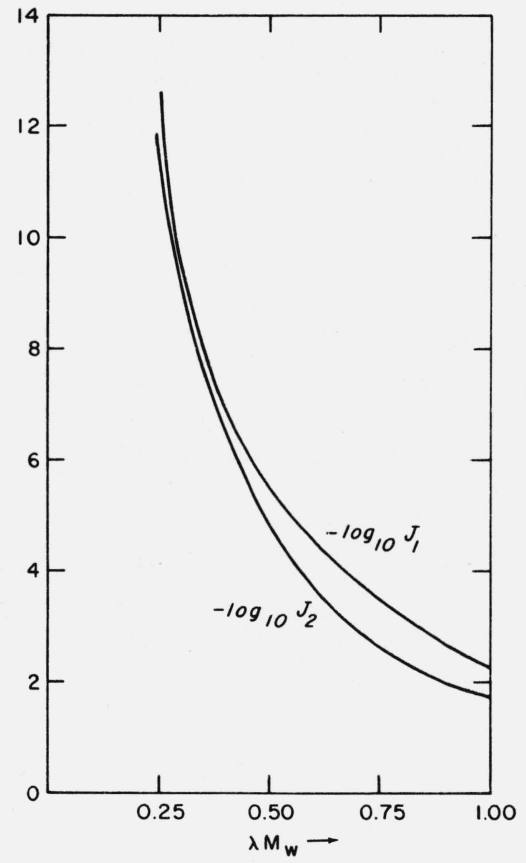

FIGURE 2. Graphs of $-\log _{10} \mathrm{~J}_{1}$ and $-\log _{10} \mathrm{~J}_{2}$ as a function of $\lambda \mathrm{M}_{\mathrm{w}}$ for Schulz distribution. $\lambda^{n}\left(\nu_{n}-\nu_{n}^{*}\right)$ can be expressed as

$$
\lambda^{n}\left(\nu_{n}-\nu_{n}^{*}\right)=\frac{\left(\lambda M_{0}\right)^{n} \sigma^{n-1}}{\sqrt{2 \pi}} \int_{\substack{\delta M_{0} \sigma}}^{\infty} u^{n-1} e^{-u^{2} / 2} d u .
$$

For odd $n$ these can be written in terms of complementary error functions and exponentials, while for even $n$ they can be written in terms of exponentials. The $J_{n}$ defined in eq (24) are expressible in the form

$$
J_{n}=\int_{\frac{2 \pi}{\lambda M_{0} \bar{\sigma}}}^{\infty} u^{n-1} e^{-u^{2} / 2} d u / \int_{0}^{\infty} u^{n-1} e^{-u^{2} / 2} d u .
$$

Specifically, the first three J's are

$$
\begin{aligned}
& J_{1}=2 \operatorname{erfc}\left(\frac{2 \pi}{\lambda M_{0} \sigma}\right) \\
& J_{2}=\exp \left(-\frac{2 \pi^{2}}{\lambda^{2} M_{0}^{2} \sigma^{2}}\right) \\
& J_{3}=J_{1}+\sqrt{2 \pi} J_{2},
\end{aligned}
$$

where $\operatorname{erfc}(x)=(2 \pi)^{-1 / 2} \int_{x}^{\infty} \exp \left(-u^{2} / 2\right) d u$.

These formulas can be simplified by noting that practical values of $\lambda M_{0} \sigma$ are of the order of 1 or less. As we have already noted, $\lambda M_{w}$ can be made of the order of 1 , and $M_{w} / M_{z}<1$ so that $\lambda M_{0}<\lambda M_{w}$. Further, for the cases where the ratios $\left(M_{z} / M_{w}\right)$ are less than approximately 10 and $\sigma<1.6$, the ratio $\frac{2 \pi}{\lambda M_{0} \sigma}$ is greater than 3 and we can approximate the complementary error function by

$$
\operatorname{erfc}\left(\frac{2 \pi}{\lambda M_{0} \sigma}\right) \sim \frac{\lambda M_{0} \sigma}{2 \pi} \exp \left(-\frac{2 \pi^{2}}{\lambda^{2} M_{0}^{2} \sigma^{2}}\right)
$$

which can be inserted in the expressions for $\lambda\left(\nu_{1}-\nu_{1}^{*}\right)$ and $J_{1}, J_{2}, J_{3}$. This procedure yields

$$
\begin{gathered}
\lambda\left(\nu_{1}-\nu_{1}^{*}\right) \sim \frac{\left(\lambda M_{0}\right)^{2} \sigma}{2 \pi} \exp \left(-\frac{2 \pi^{2}}{\lambda^{2} M_{0}^{2} \sigma^{2}}\right) \\
J_{1} \sim \frac{\lambda M_{0} \sigma}{\pi} \exp \left(-\frac{2 \pi^{2}}{\lambda^{2} M_{0}^{2} \sigma^{2}}\right) \\
J_{2} \sim \exp \left(-\frac{2 \pi^{2}}{\lambda^{2} M_{0}^{2} \sigma^{2}}\right) .
\end{gathered}
$$




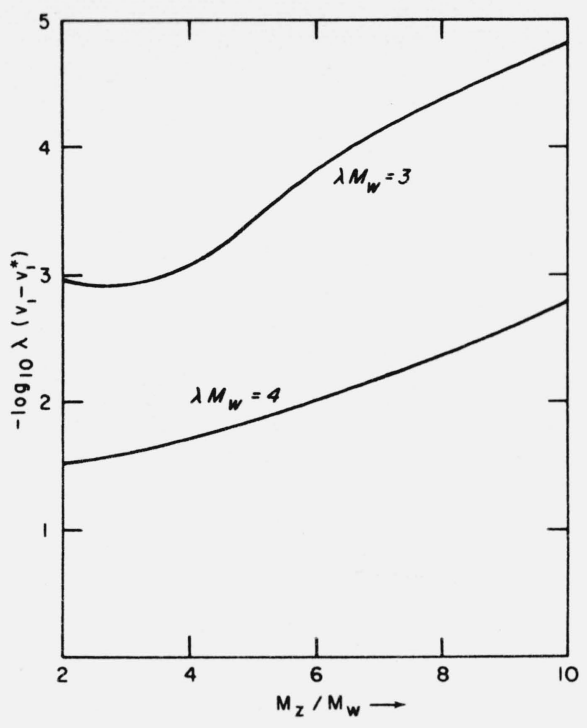

Figure 3. Graphs of $-\log _{10} \lambda\left(\nu_{1}-\nu_{1}^{*}\right)$ as a function of $\mathrm{M}_{\mathrm{z}} / \mathrm{M}_{\mathrm{w}}$ for lognormal distribution.

Figure 3 shows curves of $-\log _{10} \lambda\left(\nu_{1}-\nu_{1}^{*}\right)$ as a function of $M_{z} / M_{w}$ for $\lambda M_{w}=3$ and 4 . It can be seen that $\lambda \nu_{1}$ is very close to $\lambda \nu_{1}^{*}$ for $\lambda M_{w}=3$ and all $M_{z} / M_{w}$.
Since $\lambda M_{w}$ can be kept in the neighborhood of 1 the present technique of deriving moments from the concentration can be justified theoretically for all $M_{z} / M_{w}$ provided that the lognormal distribution is a suitable representation of the weight distribution. Calculation of the $J$ 's serves only to confirm this observation. It is of some interest to note that in contrast to the results for the Schulz distribution, the $J_{n}$ decrease when $M_{z} / M_{w}$ increases.

The two distributions assumed here are for illustrative purposes only. It is probably safe to say that if $\lambda M_{w} \leqslant 1$ the errors made in the mathematical assumptions are negligible compared to the experimental errors.

\section{References}

[1] W. D. Lansing and E. O. Kraemer, J. Am. Chem. Soc. 57, 1369 (1935).

[2] H. Fujita, Mathematical Theory of Sedimentation Analysis, (Academic Press, New York) 1962.

[3] E. T. Adams, Jr., Proc. Nat. Acad. Sci. 51, 509 (1964).

[4] H. W. Osterhoudt and J. W. Williams, J. Phys. Chem., 69, 1050 (1965).

[5] G. V. Schulz, Z. Phys. Chem. A 193, 168 (1944).

[6] M. Abramowitz and I. A. Stegun, Handbook of Mathematical Functions, p. 803, (National Bureau of Standards, W ashington, D.C.) 1964

(Paper 71Al-431) 